\title{
DESPERDÍCIO ALIMENTAR EM CONTEXTO ESCOLAR: ANÁLISE DE METODOLOGIAS DE AVALIAÇÃO PARA UMA AVALIAÇÃO CONTÍNUA
}

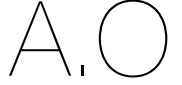

ARTIGO ORIGINAL

${ }^{1}$ CiTechCare, Centre for Innovative Care and Health Technology, School of Health Sciences, Polytechnic of Leiria, 2411-901 Leiria, Portugal

2 GeoBioTec,

GeoBioSciences,

GeoTechnologies and

GeoEngineering, Earth

Sciences Department,

Faculdade de Ciências e

Tecnologia da Universidade

NOVA de Lisboa,

2829-516 Caparica,

Portugal

3 Politécnico de Coimbra,

Escola Superior de

Tecnologia da Saúde

de Coimbra, Unidade

Científico-Pedagógica de

Dietética e Nutrição,

Rua 5 de Outubro,

São Martinho do Bispo,

3046-854 Coimbra,

Portugal

${ }^{4}$ GreenUPORTO -

Sustainable Agrifood

Production Research

Centre, Campus de

Vairão Edifício de Ciências

Agrárias (FCV2),

Rua da Agrária, n. 747 ,

4485-646 Vairão, Portuga

${ }^{5}$ LAQV-Requimte Laboratório Associado para a Química Verde,

Rua D. Manuel II,

Apartado 55142 Porto,

Portugal

`Endereço para correspondência:

João PM Lima

Escola Superior de Tecnologia da Saúde do Instituto Politécnico de Coimbra,

Rua 5 de Outubro, São Martinh do Bispo,

3046-854 Coimbra, Portuga joa.lima@estescoimbra.pt

Histórico do artigo:

Recebido a 21 de dezembro de 2020

ceite a 8 de junho de 2021

\section{FOODWASTEIN SCHOOLCONTEXT: ANALYSISOFEVALUATION METHODOLOGIES FOR A CONTINUOUS EVALUATION}

Ana Sofia Pedrosa'; Fabiana Estrada'; Matilde Silva'; Vânia Ribeiro', 2; João PM Lima', 3-5

RESUMO

INTRODUÇÃO: Em Portugal, cerca de 1 milhão de toneladas de alimentos são desperdiçados anualmente. A avaliação do desperdício alimentar nos estabelecimentos de ensino desempenha uma importante função na educação alimentar, na perspetiva nutricional, social e ética, devendo a mesma ser realizada por rotina e como forma de sensibilizar para a temática. O desperdício alimentar pode contribuir para um aporte nutricional inadequado, pelo que a sua avaliação se reveste de elevada importância.

OBJETIVOS: Comparar três metodologias de avaliação do desperdício alimentar total e por componentes numa cantina escolar do ensino superior.

METODOLOGIA: A avaliação do desperdício alimentar foi realizada nas refeições do almoço e do jantar num dia escolhido aleatoriamente. Foram utilizados os métodos de pesagem por componentes agregados, pesagem por componentes do prato e análise visual por retrato fotográfico. Foi utilizada uma amostra de 52 refeições nos métodos de análise prato-a-prato.

RESULTADOS: No método de pesagem por componente do prato obteve-se $20,5 \%$ de desperdício. No método de pesagem por componentes agregados aferiu-se um desperdício de 18,9\%. Através da análise visual do desperdício por retrato fotográfico, conclui-se que cerca de $55,8 \%$ das refeições servidas apresentavam um desperdício correspondente a $0 \%$.

CONCLUSõES: Verificou-se discrepância entre os resultados avaliados pelas metodologias por pesagem agregada e pesagem por componente no prato. Por outro lado, a metodologia da análise visual por retrato fotográfico pareceu ser uma forma menos conclusiva de análise, pois, de acordo com este método, cerca de metade das refeições não originaram restos. Face aos resultados observados, e tendo por base o procedimento de aferição do desperdício alimentar em cada uma das metodologias, o método por pesagem de componentes do prato, por amostragem, parece não ser uma metodologia tão adequada para a avaliação contínua, quando comparada com a avaliação do desperdício por componentes agregadas.

\section{PALAVRAS-CHAVE}

Desperdício alimentar, Metodologias, Alimentação coletiva

ABSTRACT

INTRODUCTION: In Portugal, about 1 million tons of food are wasted annually. The evaluation of food waste in schools plays an important role in food education, from a nutritional, social and ethical perspective, and it must be carried out routinely and as a way of raising awareness of the theme. Food waste contributes to an inadequate nutritional supply, which is why their evaluation is of high importance.

OBJECTIVES: To compare the assessment of food waste in a school canteen in higher education through the application of three methodologies, as well as to quantify food waste by component of the meal.

METHODOLOGY: The assessment of food waste was carried out at lunch and dinner meals on a randomly chosen day. Weighing methods by aggregate components, component weighing per plate and visual analysis by photographic portrait were used. A sample of 52 meals was used in the dish-by-dish analysis methods.

RESULTS: In the weighing method by component in the dish, $20.5 \%$ of waste was obtained. In the method of weighing by aggregate components, a waste of $18,9 \%$ was measured. Regarding the method of visual analysis of waste by photographic portrait, it is concluded that about $55.8 \%$ of the meals served had $0 \%$ waste.

CONCLUSIONS: There was a poor agreement between the results evaluated by the methodologies by aggregate weighing and weighing by component in the dish, verifying an apparent overestimation of the index of remains observed by the weighing method in the dish. On the other hand, the methodology of visual analysis by photographic portrait constitutes a less conclusive form of analysis, because, according to this method, about half of the meal did not originate remains. Thus, it is concluded that the method for weighing components in the dish, by sampling, should not be used as a routine methodology in the assessment of waste, opting for the aggregate component assessment.

KEYWORDS

Food waste, Methodologies, Food service 


\section{INTRODUÇÃO}

As atuais tendências de aumento populacional, o crescimento da procura alimentar e a consequente necessidade de utilização de recursos naturais de forma desmedida e inconsequente destaca a necessidade da adoção de um padrão de consumo alimentar mais sustentável (1, 2), meta alinhada com os Objetivos do Desenvolvimento Sustentável 2030 (3).

A par da promoção da sustentabilidade do consumo encontra-se a necessidade de redução do desperdício alimentar, que tem sido um tema de discussão frequentemente associado a aspetos sociais, ambientais e de controlo de custos, em diversos países e por diversas organizações internacionais (4-6). De acordo com o relatório do Parlamento Europeu, não existe uma definição consensual a nível da União Europeia, contudo no presente documento foi definido como "um produto cultivado ou parte dele, pescado ou transformado para consumo humano, que poderia ter sido consumido se tivesse sido tratado ou armazenado de forma diferente"(7).

Num serviço de restauração coletiva é fundamental distinguir sobras de restos (8). Assim, "sobras" são alimentos que são cozinhados e não chegam a ser distribuídos, podendo ser reaproveitadas desde que cumpram todas as normas de higiene e segurança alimentar durante a sua conservação. Em contrapartida, "restos" são os alimentos que são distribuídos, mas não são consumidos, não sendo possível fazer o seu reaproveitamento (9-11). A sua existência poderá contribuir para um aporte nutricional inadequado, pelo que o estudo do desperdício alimentar se reveste de grande importância (12). Um estudo recentemente publicado pelo United Nations Environment Programme refere que foram gerados 931 milhões de toneladas de desperdício alimentar relativamente ao ano de 2019, sendo que grande parte destas perdas advêm das habitações/domicílios com $61 \%$ de desperdício, 26\% de serviços de alimentação e 13\% do retalho alimentar (13).

Na Europa, 88 milhões de toneladas de alimentos são desperdiçados anualmente, ou seja, 179 quilogramas (kg)/per capita o que equivale a um custo de 143 biliões de euros (14).

Em Portugal, o Projeto de Estudo e Reflexão sobre o Desperdício Alimentar (PERDA) estimou que 17\% das partes comestíveis dos alimentos produzidos para consumo humano são desperdiçadas, correspondendo aproximadamente a um milhão de toneladas anualmente (15).

A Comissão Nacional do Combate ao Desperdício Alimentar (CNCDA), foi criada com o objetivo de combater o desperdício alimentar, numa responsabilidade partilhada com o produtor ao consumidor, define a implementação de várias medidas, centradas em três pontos chave: prevenção, através da sensibilização das populações, autarquias e comunidade escolar para o problema do desperdício alimentar; monitorização do desperdício alimentar ao longo das diferentes fases da cadeia alimentar; redução do desperdício alimentar através da simplificação de barreiras administrativas, cooperação entre agentes e inovação de práticas para redução do desperdício (16).

A CNCDA estabeleceu como necessidade uma maior intervenção das instituições escolares, nomeadamente ao nível universitário de modo a promover o conhecimento, a inovação e o empreendedorismo ao nível do desperdício alimentar, bem como a criação de sinergias entre as universidades que permitam a monitorização e investigação neste âmbito (17)

Vários fatores podem influenciar o desperdício, nomeadamente o planeamento inadequado das refeições, as capitações oferecidas, o aspeto visual, as preferências alimentares dos consumidores, o apetite e a formação inadequada dos trabalhadores, entre outros, pelo que é difícil a sua eliminação por completo (18-21).

É conhecido que o planeamento adequado das refeições e a avaliação contínua do desperdício alimentar permitirá aos agentes responsáveis (nutricionista, cozinheiros e ajudantes de cozinha) pela produção de refeições, minimizar a sua ocorrência (22). Contudo, pese embora, exista entendimento generalizado acerca da importância da aferição do desperdício alimentar para um melhor controlo e sua minimização, não existe consenso técnico-científico acerca do método mais adequado para a realização da sua medição em diferentes contextos, também motivado pela necessidade de adequar a avaliação às condições laborais e ao grau de precisão requerido (23).

Face ao exposto, este trabalho pretende ser um contributo para a aferição das metodologias mais adequadas para a avaliação contínua do desperdício no contexto do fornecimento de refeições em unidades de alimentação do ensino superior.

\section{OBJETIVOS}

Comparar três metodologias de avaliação do desperdício alimentar numa cantina do ensino superior, tendo em vista aferir qual a metodologia de avaliação mais eficiente, capaz de induzir uma menor percentagem de erros nos resultados para ser utilizada na avaliação contínua do desperdício alimentar numa unidade de alimentação de uma instituição de ensino superior público.

\section{METODOLOGIA}

O presente estudo foi realizado numa unidade de alimentação de uma instituição de ensino superior público, constituído por uma cantina e um snack-bar, tendo sido a mesma selecionada por ser a que entre as congéneres da instituição apresentava um maior volume de refeições anual. A cantina serve, cerca de 62.000 refeições anualmente, com maior predominância no período de almoço, enquanto o snack-bar produz uma média de 54.500 refeições anualmente, também em maior quantidade no período de almoço. Na Tabela 1 é descrita a ementa do dia em análise. Optou-se por analisar apenas o prato principal, pois a sopa e salada eram de caráter opcional. Não foi considerado o prato vegetariano devido à baixa adesão relativa a esta opção e expectável maior sensibilidade por parte de quem o consome para o tema da sustentabilidade (24).

Na cantina são disponibilizados diariamente 3 pratos principais diferentes, nomeadamente um prato em que a componente proteica é carne, um prato contendo a componente proteica de peixe e um prato vegetariano. Os menus são constituídos por sopa, prato principal (constituído por uma componente fornecedora de hidratos de carbono, uma componente fornecedora de proteína e um acompanhamento de hortícolas), pão, sobremesa e bebida (sumo ou água), sendo o preço da refeição completa 2.65€ (mais $0.25 €$ se adquirida no próprio dia). No snack-bar apenas existe uma opção, que geralmente alterna entre o período do almoço e do jantar, um prato contendo a componente proteica de peixe ou carne alternadamente. O preço da refeição é pago consoante o que é selecionado na linha de distribuição, não havendo um preço fixo pré-estabelecido. Adicionalmente, existe à disposição dos utilizadores um buffet de saladas, composto por alface, cenoura, tomate, milho, couve-roxa e cebola. Foram considerados pratos compostos todos os pratos onde a componente proteica é confecionada juntamente com a componente fornecedora de hidratos de carbono (25). Por guarnição entende-se o conjunto de alimentos que são servidos em conjunto com a componente fornecedora de proteína (26), isto é, a componente fornecedora de hidratos de carbono e o acompanhamento de hortícolas. 
Ementa relativa ao período do almoço e do jantar na unidade de alimentação

\begin{tabular}{|c|c|c|c|}
\hline & PRATOS & ALMOço & JANTAR \\
\hline \multirow{2}{*}{ Cantina } & Carne & $\begin{array}{l}\text { Carne de porco assada com arroz de passas } \\
\text { Alergénios: Sulfitos, pode conter vestígios de glúten }\end{array}$ & $\begin{array}{l}\text { Bife à cervejaria com arroz e batata frita } \\
\text { Alergénios: Glúten, leite }\end{array}$ \\
\hline & Peixe & $\begin{array}{l}\text { Maruca de cebolada com puré de batata } \\
\text { Alergénios: Peixe, ovo, sulfitos }\end{array}$ & $\begin{array}{l}\text { Lulas guisadas com batata, cenoura e ervilhas } \\
\text { Alergénios: Moluscos, sulfitos }\end{array}$ \\
\hline \multirow{2}{*}{ Snack-bar } & Carne & & $\begin{array}{l}\text { Massa à Carbonara } \\
\text { Alergénios: Sulfitos, leite, ovo, glúten, vestígios de } \\
\text { aipo, soja, peixe ou marisco }\end{array}$ \\
\hline & Peixe & $\begin{array}{l}\text { Bacalhau da avó com batata frita às rodelas e legumes } \\
\text { cozidos } \\
\text { Alergénios: Peixe, glúten }\end{array}$ & \\
\hline
\end{tabular}

A recolha de dados foi autorizada institucionalmente, e todos os intervenientes no estudo utilizaram material de proteção adequado, que consistiu em toucas para o cabelo, luvas, batas e pés descartáveis. As metodologias de avaliação do desperdício alimentar testadas foram as seguintes: pesagem por componentes agregados $(8,27$, 28); pesagem por componentes no prato (29-31); e análise visual do desperdício por retrato fotográfico (32-37).

\section{Pesagem por Componentes Agregados}

Todas as cubas com os alimentos confecionados foram pesadas por componente fornecedora de hidratos de carbono e componente fornecedora de proteína a priori e a posteriori da refeição, de forma a quantificar as sobras. As cubas utilizadas foram, previamente, pesadas sem conteúdo para, posteriormente, anular o peso das mesmas. No final da refeição os restos alimentares de cada prato foram divididos novamente por componente e colocados em cubas vazias. Estas cubas foram posteriormente pesadas, de forma a obter o peso dos restos, descontando o peso da mesmas $(8,27,28)$. Esta metodologia foi replicada também no snack-bar e foram utilizadas duas balanças Soehnle CW 774 (Max: 20kg, dd=5g).

\section{Pesagem por Componentes no Prato}

Foi definida uma amostra por conveniência de trinta refeições, sendo que quinze pratos analisados corresponderam aos pratos contendo componente proteica de carne e os restantes quinze aos pratos contendo componente proteica de peixe. Os pratos, devidamente diferenciados e numerados, foram pesados sem conteúdo e, posteriormente, pesados, de forma individualizada, por componente agregado. No final da refeição, estes pratos foram novamente pesados por componente, de forma a quantificar os restos $(29,30)$. No período de almoço apenas foi possível a análise de 7 pratos da componente fornecedora de proteína de pescado, devido à baixa adesão. Nesta metodologia foi utilizada uma balança EB Series OHAUS (Max: 12,5kg, dd= $5 \mathrm{~g}$ ).

\section{Análise Visual do Desperdício por Retrato Fotográfico}

Todos os pratos numerados no método anterior foram fotografados no início e no fim da refeição. Nesta metodologia foi utilizada uma máquina fotográfica Cannon $600 \mathrm{D}$ com o auxílio de um tripé a cerca de 1,5 metros do chão, tendo sido a câmara colocada a um ângulo de $45^{\circ}$ e direcionada para a mesa onde se encontravam os pratos. Os pratos selecionados aleatoriamente e distribuídos pelos consumidores ao acaso, foram numerados de 1 a 30 na parte inferior de forma que o utilizador não percebesse que estava a ser avaliado, e como tal não enviesasse o seu consumo. Os tabuleiros identificados aquando da recolha foram reservados para serem posteriormente alvos de comparação relativamente ao que tinha sido servido. As fotografias foram analisadas pelas investigadoras do estudo e, posteriormente comparadas com as imagens relativas a uma escala de 5 pontos utilizada por Hanks (23, 32, 34-37). De referir que não houve recolha de qualquer informação relativa aos indivíduos que consumiram os pratos identificados.

\section{Metodologia Utilizada na Quantificação do Desperdício}

O peso dos alimentos produzidos foi calculado através da subtração do peso de todas as cubas com alimentos prontos a servir pelo peso médio das cubas vazias. A quantidade de alimentos distribuídos calculou-se através do peso total de alimentos produzidos subtraindo o peso das sobras (10).

Para o cálculo da componente proteica foi considerada apenas a parte edível, tendo por base as percentagens da parte edível descritas na Tabela de Composição de Alimentos (38).

Para a avaliação do desperdício alimentar foram aplicadas as seguintes fórmulas (39):

$\%$ Índice de Restos $=\frac{\text { Peso dos Restos }}{\text { Peso de alimentos distribuidos }} \times 100$

$\%$ Índice de Sobras $=\frac{\text { Peso das Sobras }}{\text { Peso de alimentos produzidos }} \times 100$

$\%$ de Desperdício $=\underline{\text { Peso total de desperdício (Peso das Sobras }+ \text { Peso dos Restos) }} \times 100$ Peso de alimentos produzidos

Consumo per capita por refeição =

Peso total de alimentos produzidos - Peso total do desperdício Número de refeiç̧ôes servidas

Desperdício médio per capita $=\frac{\text { Peso total do desperdício }}{\text { Número de refeiçōes senvidas }}$

A análise foi igualmente realizada tendo por base o número de refeições que se poderia servir com os alimentos desperdiçados, obtida através da divisão do peso dos alimentos desperdiçados pela quantidade de alimentos servidos por refeição $(28,40)$.

\section{RESULTADOS}

No dia em análise foram confecionadas um total de 982 refeições. $\mathrm{Na}$ Tabela 2 encontra-se a descrição do número de refeições servidas por tipologia de componente proteica e refeição.

\section{Pesagem por Componentes Agregados}

No dia da avaliação foram produzidos $365 \mathrm{~kg}$ de alimentos, sendo que $69 \mathrm{~kg}$ de alimentos foram desperdiçados, $22,5 \mathrm{~kg}$ sob a forma de sobras e $46,5 \mathrm{~kg}$ de restos. Tendo em conta o número de refeições 
servidas (982 refeições) procedeu-se ao cálculo da quantidade de alimentos servida por cada refeição, cerca de $0,35 \mathrm{~kg}$, tendo em conta a quantidade de alimento servido (365 kg - 22,5 kg). Assim, verifica-se que foram desprezadas cerca de 198 refeições. Este raciocínio pode ser aplicado ao valor das sobras, alimentos produzidos não distribuídos, concluindo que cerca de 65 refeições poderiam ter sido servidas. Em tese, pode aferir-se que o consumo per capita por refeição foi em média $0,301 \mathrm{~kg}$, resultando, por sua vez, num desperdício médio per capita por refeição de $7 \%$.

Atendendo aos resultados obtidos, o desperdício total de alimentos do dia foi de 18,9\%, que é considerado "inaceitável" tendo por base a classificação do desperdício alimentar de Aragão (41). O índice de restos foi de 13,6 \% enquanto o índice de sobras foi de 6,2 \%.

Considerando o desperdício total do dia, o momento da refeição que apresentou maior desperdício foi o período do almoço, sendo que $23,6 \%$ dos alimentos foram desperdiçados. Em oposição, no jantar o desperdício foi menor, uma vez que cerca de $11,1 \%$ foram desperdiçados.

Na cantina, o desperdício no prato de carne no período do almoço foi de $23,9 \%$ e, no período do jantar foi de $14,9 \%$. Comparativamente, o prato de peixe apresentou $28,8 \%$ de desperdício, no almoço e 17,4\% ao jantar. Como tal, ao almoço foram desperdiçados $24,2 \%$ de alimentos e ao jantar $15,4 \%$, tendo em consideração as sobras e os restos.

No snack-bar, a ementa do almoço foi constituída por um prato de carne e um prato de peixe, no entanto, o jantar apresentou apenas um prato composto. No almoço, o desperdício apresentado no prato de carne foi de $20,5 \%$, enquanto no prato de peixe foi de $25 \%$. A ementa do jantar obteve um desperdício de $5,7 \%$. Em síntese, verificou-se que o desperdício no almoço foi de $22,6 \%$, enquanto no jantar foi de 5,7\%. Na Tabela 3 encontram-se discriminados os resultados relativos à avaliação de sobras, restos e desperdício total, na cantina e snack-bar, por refeição e componente proteica da mesma.

\section{Pesagem por Componente no Prato}

O método de pesagem de componente por prato foi realizado ao almoço e jantar. Nesta metodologia foram analisados 52 pratos, 22 pratos no período do almoço, sendo que 15 pratos corresponderam a pratos contendo como fonte proteica a carne e 7 pratos contendo como fonte proteica o peixe e, no período do jantar foram analisadas 30 refeições, sendo que 15 refeições analisadas continham como componente proteica a carne e 15 refeições continham como componente proteica o peixe. Na cantina, ao almoço, nos pratos de carne, o componente de proteína apresentou um desperdício de $25,4 \%$ e a componente de guarnição de $30,8 \%$, perfazendo um total de $28,4 \%$ de desperdício. Relativamente aos pratos de peixe, o componente fornecedor de proteína apresentou de desperdício 35,5\%, enquanto o componente de guarnição 3,9\%. O desperdício total nos pratos de peixe foi de $17,2 \%$.

No período do jantar, na cantina, na análise dos pratos de carne, desperdiçou-se $16,1 \%$ do componente fornecedor de proteína e $30,4 \%$ do componente de guarnição, perfazendo um total de 23,6\% de desperdício no respetivo prato. O prato de peixe apresentava a tipologia de prato composto, apresentando 15,2\% como desperdício total (6,9 kg produzidos e 1,0 kg de restos).

Na pesagem por componente por prato, o desperdício apresentou mais expressão no prato de carne, no período do almoço. No período do jantar, o prato de carne foi o que obteve, igualmente, maior desperdício. Os resultados são apresentados na Tabela 4.

Considerando o desperdício total dos pratos em ambas as refeições por componente, constatou-se que foi desperdiçada $23,9 \%$ do componente proteico produzida e $23,6 \%$ da guarnição, perfazendo 20,5\% das refeições desperdiçadas.

Considerando o desperdício total do dia, o momento da refeição que apresentou maior desperdício foi o período do almoço (23,6\% vs. $11,1 \%)$. Ao avaliar o desperdício por componentes no almoço e no jantar,

\section{Tabela 2}

Ementa relativa ao período do almoço e do jantar na unidade de alimentação

\begin{tabular}{|c|c|c|c|}
\hline & ALMOço & JANTAR & TOTAL \\
\hline \multirow{2}{*}{ Cantina } & Carne - 412 & Carne - 161 & \multirow{2}{*}{611} \\
\hline & Peixe - 13 & Peixe -25 & \\
\hline \multirow{2}{*}{ Snack-bar } & Carne - 140 & \multirow{2}{*}{ Prato único - 144} & \multirow{2}{*}{371} \\
\hline & Peixe - 87 & & \\
\hline TOTAL & 652 & 330 & 982 \\
\hline
\end{tabular}

\section{Tabela 3}

Caracterização das sobras, restos e desperdício total, na cantina e snack-bar, por refeição e componente proteica da mesma

\begin{tabular}{|c|c|c|c|c|c|}
\hline & & \multicolumn{2}{|c|}{ CANTINA } & \multicolumn{2}{|c|}{ SNACK-BAR } \\
\hline & & ALMOÇO & JANTAR & ALMOço & JANTAR \\
\hline \multirow{4}{*}{ PRATO DE CARNE } & Produzido (kg) & 136,3 & 61,8 & 43,9 & 60,6 \\
\hline & Sobras (kg) & 11,8 & 1,7 & 3,3 & \\
\hline & Restos (kg) & 20,8 & 7,5 & 5,7 & 0,0 \\
\hline & Desperdício (\%) & 23,9 & 14,9 & 20,5 & \\
\hline \multirow{4}{*}{ PRATO DE PEIXE } & Produzido (kg) & 8,1 & 15,5 & 38,8 & 3,5 \\
\hline & Sobras (kg) & 1,0 & 0,0 & 4,8 & \\
\hline & Restos (kg) & 1,4 & 2,7 & 4,9 & 5,7 \\
\hline & Desperdício (\%) & 28,9 & 17,4 & 25,0 & \\
\hline \multirow{4}{*}{ TOTAL } & Produzido $(\mathrm{kg})$ & 144,4 & 77,3 & 82,7 & 60,6 \\
\hline & Sobras (kg) & 12,8 & 1,7 & 8,1 & 0,0 \\
\hline & Restos (kg) & 22,2 & 10,2 & 10,6 & 0,0 \\
\hline & Desperdício (\%) & 24,2 & 15,4 & 22,6 & 5,7 \\
\hline
\end{tabular}


constata-se um maior desperdício no componente proteico sendo que no período do almoço desperdiçou-se o dobro de alimentos.

\section{Análise Visual do Desperdício por Retrato Fotográfico}

No método de análise visual por retrato fotográfico analisou-se, de forma crítica, os restos provenientes dos pratos que foram alvo de estudo pelo método de pesagem por componente no prato. Nesta metodologia foram analisados 52 pratos, 22 pratos no período do almoço, sendo que 15 pratos corresponderam a pratos contendo como fonte proteica a carne e 7 pratos contendo como fonte proteica o peixe e, no período do jantar foram analisadas 30 refeições, sendo que 15 refeições analisadas continham como componente proteica a carne e 15 refeições continham como componente proteica o peixe. De acordo com o estudo de Hanks, 2014 estabeleceu-se uma escala de cinco pontos para a avaliação da quantificação do desperdício através do visionamento do prato no final da refeição (23), descrita na Tabela 5

Com base na escala estabelecida anteriormente, a análise dos dados relativos ao desperdício gerados pelo método de análise visual por retrato fotográfico é apresentada no Gráfico 1. Assim, é

\section{Tabela 4}

Desperdício alimentar por componente da refeição e refeição

\begin{tabular}{|c|c|c|c|c|c|}
\hline & & \multicolumn{2}{|c|}{ CANTINA } & \multicolumn{2}{|c|}{ SNACK-BAR } \\
\hline & & PRATO DE CARNE & PRATO DE PEIXE & PRATO DE CARNE & PRATO DE PEIXE \\
\hline \multirow{3}{*}{$\begin{array}{l}\text { COMPONENTE } \\
\text { PROTEICA }\end{array}$} & Produzido (kg) & 1,8 & 1,2 & 2,1 & NA \\
\hline & Restos (kg) & 0,4 & 0,4 & 0,3 & NA \\
\hline & Desperdício (\%) & 25,4 & 35,5 & 16,1 & NA \\
\hline \multirow{3}{*}{ GUARNIÇÃO } & Produzido (kg) & 2,2 & 1,6 & 2,3 & NA \\
\hline & Restos (kg) & 0,7 & 0,1 & 0,7 & NA \\
\hline & Desperdício (\%) & 30,8 & 3,9 & 30,4 & NA \\
\hline \multirow{3}{*}{ TOTAL } & Produzido (kg) & 3,9 & 2,76 & 4,4 & NA \\
\hline & Restos (kg) & 1,1 & 0,48 & 1,0 & NA \\
\hline & Desperdício (\%) & 28,4 & 17,2 & 23,6 & NA \\
\hline
\end{tabular}

\section{Tabela 5}

Exemplificação da avaliação do desperdício alimentar com recurso ao método de estimativa visual por retrato fotográfico

\% DESPERDÍCIO CORRESPONDÊNCIA NO PRATO

$100 \%$

desperdício total

\section{FIGURA}
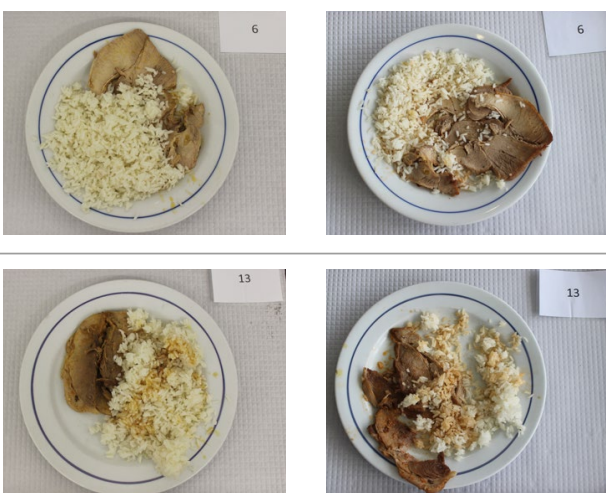

$75 \%$

$3 / 4$ do prato cheio
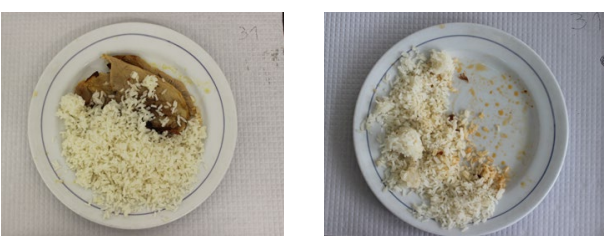

$50 \%$

$1 / 2$ do prato cheio
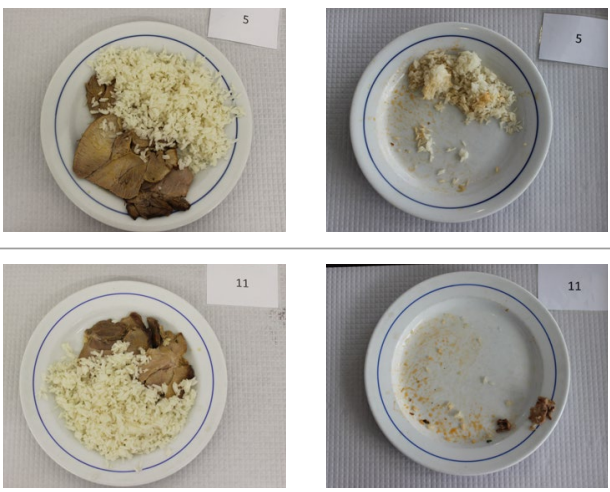
possível constatar que, por meio da análise, 10 pratos apresentavam desperdício nulo, 8 pratos desperdiçaram cerca de 25\%, 1 prato continha 50\% dos restos, 2 pratos obtiveram um desperdício de $75 \%$ e apenas um prato apresentava desperdício de 100\% (Gráfico 1). Ao jantar verificou-se que 19 pratos apresentaram um desperdício nulo por cada refeição, 9 pratos demonstraram um desperdício de 25\%, e em 2 pratos analisados foi desperdiçado metade da refeição. Não houve desperdício nos itens correspondentes ao desperdício de 100\% e 75\% (Gráfico 2).

Tendo em conta os resultados obtidos nesta metodologia, é possível afirmar que o desperdício foi avaliado como moderado nos pratos analisados, uma vez que a quantidade desperdiçada corresponde, maioritariamente, a uma quantidade desperdiçada nula ou de $25 \%$. Ao jantar, o desperdício foi relativamente menor, visto que os pratos que não originaram desperdício representam grande parte da amostra dos pratos analisados.

\section{DISCUSSÃO DOS RESULTADOS}

As cantinas universitárias são responsáveis por uma produção considerável de resíduos alimentares, sendo um dos locais a destacar para a educação sobre o desperdício de alimentos (22, 42-44). O desperdício de alimentos nas cantinas pode ser minimizado através da educação de alunos e funcionários para a alteração dos comportamentos e desenvolvimento de estratégias de sensibilização $(22,40,45)$.

Os possíveis fatores envolvidos no desperdício no domínio das cantinas incluem a inadequação das porções servidas; a consciência,

\section{Gráfico 1}

Gráfico do desperdício alimentar no período de almoço

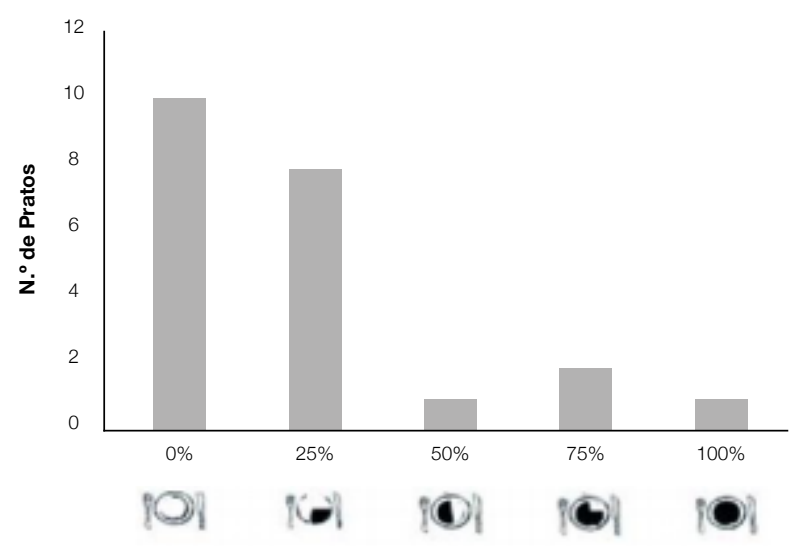

Gráfico 2

Gráfico do desperdício alimentar no período de jantar

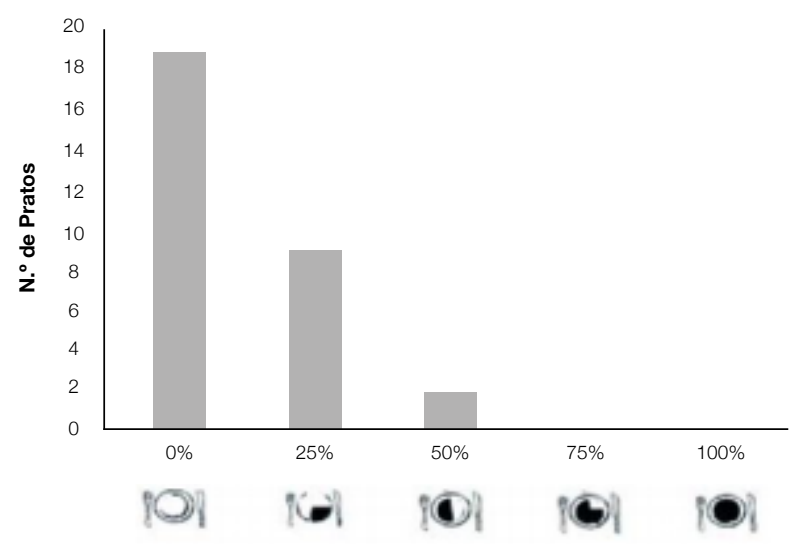

conhecimento e sensibilização face à temática do desperdício, as preferências alimentares, a qualidade e aparência da refeição, o planeamento e a logística da refeição (11, 20, 44). Segundo Garrone et al., 2014, realizar uma auditoria regular aos resíduos quer na preparação quer nas zonas de distribuição, é o primeiro passo para reduzir o desperdício de alimentos (46).

O desperdício nas unidades de alimentação em análise é referido como "inaceitável", uma vez que apresenta um valor igual ou superior a 10\%. A implementação de programas de sensibilização para a comunidade académica relacionados com a importância da redução do desperdício alimentar poderão ser preponderantes no combate a esta problemática.

Os níveis de desperdício alimentar identificados no presente estudo foram concordantes com os observados por outros autores em contextos semelhantes $(8,11,28,43)$.

De referir que grande parte dos trabalhos identificados na revisão da literatura, especialmente em Portugal (27, 30, 40, 47), dizem respeito a avaliações de desperdício realizadas em cantinas escolares de escolas básicas ou secundárias e não no contexto do ensino superior. Apenas um estudo realizado numa universidade Portuguesa que concluiu que eram desperdiçados sob a forma de sobras $0,080 \mathrm{~kg}$ e sobre a forma de restos $0,200 \mathrm{~kg}$ o que foi de encontro ao verificado neste estudo $0,061 \mathrm{~kg} \mathrm{e} \mathrm{0,200} \mathrm{kg}$, respetivamente. Diferendo apenas na quantidade de alimentos distribuído obtendo 0,940 kg e no presente estudo, 0,350 $\mathrm{kg}$ o que pode ter influenciado a quantidade de restos (11).

A presença de restos, neste caso em concreto poderá ser explicado através do enviesamento de capitações, pois as quantidades fornecidas eram acima do necessário. Por outro lado, a falta de planeamento relativamente à quantidade de refeições a produzir gerou a presença de sobras, uma vez que grande parte das refeições não eram reservadas nem solicitadas antecipadamente.

Relativamente às metodologias de avaliação do desperdício alimentar utilizadas, este trabalho pretendia identificar qual a que poderia ser utilizada de forma contínua na avaliação do desperdício alimentar numa cantina do ensino superior. Tendo em conta os resultados observados, os métodos de avaliação por pesagem por componentes agregados e no prato apresentou resultados sobreponíveis, razão pela qual num contexto de rotina e como forma de não enviesar o consumo do utilizador poderá ser utilizada a primeira. Metodologia semelhante foi utilizada por vários autores $(8,27-29,31)$. Relativamente à análise visual do desperdício por retrato fotográfico, com classificação de acordo com a escala de 5 pontos utilizada por Hanks (2014) (23), o mesmo demonstrou não ser sensível face ao número de pratos que classificou como 0\% desperdício. Como tal, esta metodologia parece subestimar o desperdício alimentar. Resultados diferentes foram observados em outro estudo, onde foi utilizada a escala de Comstock de 6 pontos, que identificou que a análise visual parecia sobrestimar a quantificação do desperdício alimentar (48). Trabalhos futuros poderão quantificar o desperdício alimentar com recurso a manual de quantificação de alimentos (49) tornando a aferição e análise mais criteriosa, pese embora o facto de ser uma metodologia mais morosa possa ser vista como um inconveniente.

De acordo com a literatura, a aparência visual e a quantidade de alimentos podem afetar a capacidade de um observador estimar o seu peso com precisão (50). Porções menores têm habitualmente menor probabilidade de serem estimadas com precisão do que porções maiores e poderiam resultar numa interpretação enganosa de grupos de alimentos não significativos $(50,51)$. Outros autores concluíram que a precisão da estimativa não foi afetada pela aparência do alimento, consistência e recipiente da porção, determinando que a 
estimativa visual parece ser um substituto adequado para o método de pesagem (52). Características do avaliador, como sua motivação e nível de educação em nutrição, também podem afetar a precisão da estimativa visual (52). A familiaridade com a porção de alimentos e o treino intensivo também aumentaram a precisão da estimativa de desperdício subsequente $(50,51,53-56)$. Um estudo que comparou várias metodologias de quantificação de desperdício alimentar verificou que as técnicas de estimativa visual levam aproximadamente um quinto do tempo exigido pelo método de pesagem (23).

O método de análise visual por retrato fotográfico apresenta algumas fragilidades por ser mais difícil de interpretar e os resultados dos grupos de alimentos agregados podem apresentar falta de suporte para o método visual (51), bem como o facto das avaliações não serem feitas em proporções exatas $(23,54)$. Além disso, o menor custo e a maior conveniência deste método em relação ao método de pesagem foram apontados por outros investigadores para apoiar a sua utilização na avaliação do desperdício alimentar $(56,57)$.

\section{CONCLUSÕES}

No método de pesagem por componente no prato obteve-se 20,5\% de desperdício. No método de pesagem por componentes agregadas verificou-se um desperdício de 18,9\%, enquanto utilizando o método da análise visual do desperdício por retrato fotográfico, conclui-se que cerca de 55, 8\% das refeições servidas não apresentam desperdício. Verificou-se discrepância entre os resultados avaliados pelas metodologias por pesagem agregada e pesagem por componente no prato. Por outro lado, a metodologia da análise visual por retrato fotográfico pareceu ser uma forma menos conclusiva de análise, pois, de acordo com este método, cerca de metade das refeições não originaram restos. Face aos resultados observados, e tendo por base o procedimento de aferição do desperdício alimentar em cada uma das metodologias, o método por pesagem de componentes do prato, por amostragem, parece não ser uma metodologia tão adequada para a avaliação contínua, quando comparada com a avaliação do desperdício por componentes agregadas.

\section{REFERÊNCIAS BIBLIOGRÁFICAS}

1. Comissão Europeia. Para uma economia circular: programa para acabar com os resíduos na Europa. Comunicação da Comissão ao Parlamento Europeu, ao Conselho, ao Comité Económico e Social Europeu e ao Comité das Regiões. 2014.

2. Comissão Europeia. Fechar o ciclo - plano de ação da UE para a economia circular. Comunicação da Comissão ao Parlamento Europeu, ao Conselho, ao Comité Económico e Social Europeu e ao Comité das Regiões. 2015.

3. United Nations. Transforming our word:The 2030 Agenda for sustainable development. 2016;12-4.

4. Gjerris M, Gaiani S. Household food waste in Nordic countries: Estimations and ethical implications. Etikk i praksis Nord J Appl Ethics. 2013;7(1):6-23.

5. Parfitt J, Barthel M, Macnaughton S. Food waste within food supply chains: quantification and potential for change to 2050. Philos Trans R Soc B Biol Sci. 2010 Sep;365(1554):3065-81.

6. Food and Agriculture Organization of the United Nations. Global food losses and food waste. 2011.

7. Comissão Nacional de Combate ao Desperdício Alimentar. Relatório Especial - Luta contra o desperdício alimentar: uma oportunidade para a UE melhorar a eficiência dos recursos na cadeia de abastecimento alimentar. 2016.

8. Carvalho JG, Lima JPM, Rocha AMCN da. Desperdício Alimentar E Satisfação Do Consumidor Com O Serviço De Alimentação Da Escola De Hotelaria E Turismo De Coimbra, Portugal. DEMETRA Aliment Nutr Saúde [Internet]. 2015;10(2):405-18. Available from: http://www.e-publicacoes.uerj.br/index.php/demetra/article/view/15423.

9. Abreu E. Gestão de unidades de alimentação e nutrição: um modo de fazer. 2007.
10. Müller PC. Avaliação de desperdício de alimentos na distribuição do almoço servido para os funcionários de um hospital público de Porto Alegre - RS. 2008.

11. Ferreira M, Martins ML, Rocha A. Food waste as an index of foodservice quality. $\mathrm{Br}$ Food J. 2012;115(11):1628-37.

12. Palmisano G, Bottalico F, Bilali H El, Cardone G, Capone R. Chapter 10 - Food losses and waste in the context of sustainable food and nutrition security. In: Food Security and Nutrition [Internet]. Academic Press; 2021. p. 235-55. Available from: https://www. sciencedirect.com/science/article/pii/B9780128205211000101?via\%3Dihub.

13. United Nations Environment Programme. Food Waste Index. 2021. 3-99 p.

14. Stenmarck Å, Jensen C, Quested T, Moates G, Cseh B, Juul S, et al. FUSIONS Estimates of European food waste levels. Fusions. 2016. 1-80 p.

15. Baptista P, Campos I, Pires I, Vaz S. Do Campo ao Garfo. 2012.

16. Comissão Nacional de Combate ao Desperdício Alimentar. Estratégia Nacional e Plano de Ação de Combate ao Desperdício Alimentar [Internet]. 2017 [cited 2018 Jan 5]. Available from: http://www.gpp.pt/images/MaisGPP/Iniciativas/CNCDA/ENCDA.pdf. 17. Conselho de Ministros. Resolução do Conselho de Ministros n.046/2018. Diário da República, 822018 p. 1708-20.

18. Buzby JC, Guthrie JF. Plate Waste in School Nutrition Programs: Final Report to Congress. US Food Assist Nutr Res Progr. 2002;(March):1-20.

19. Nonino-Borges CB, Rabito El, Da Silva K, Ferraz CA, Chiarello PG, Dos Santos JS, et al. Food wastage in a hospital. Rev Nutr. 2006;19(3):349-56.

20. European Commission, Report T. Preparatory Study on Food Waste Across Eu 27 [Internet]. Vol. 33, October. 2010. 17 p. Available from: http://ec.europa.eu/environment/ eussd/pdf/bio_foodwaste_report.pdf.

21. Boschini M, Falasconi L, Cicatiello C, Franco S. Why the waste? A large-scale study on the causes of food waste at school canteens. J Clean Prod. 2020 Feb;246:118994. 22. Derqui B, Fernandez V. The opportunity of tracking food waste in school canteens: Guidelines for self-assessment. Waste Manag. 2017.

23. Hanks AS, Wansink B, Just DR. Reliability and accuracy of real-time visualization techniques for measuring school cafeteria tray waste: Validating the quarter-waste method. J Acad Nutr Diet [Internet]. 2014;114(3):470-4. Available from: http://dx.doi.org/10.1016/j.jand.2013.08.013.

24. Kim MJ, Hall CM, Kim DK. Predicting environmentally friendly eating out behavior by value-attitude-behavior theory: does being vegetarian reduce food waste? J Sustain Tour [Internet]. 2020;28(6):797-815. Available from: https://doi.org/10.1080/0966958 2.2019.1705461.

25. Marques H, Oliveira B, Graça M, Fernandes E, Silva S. Guidelines para a elaboração de um plano de ementas. Porto: Associação Portuguesa dos Nutricionistas; 2007.

26. Gomes S, Ávila H, Oliveira B, Franchini B. Para Refeições Em Meio Escolar: 2015. $92 \mathrm{p}$.

27. Familiar D, Rocha A. Estudo do Desperdício Alimentar em Escolas do 10 Ciclo do Ensino Básico e Jardim de Infância. Spcna. 2013;19:2013.

28. Ribeiro J, Rocha A. Economic impact of food waste in a school centre. Acta Port Nutr. 2019; 19: 36-41.

29. Cohen JFW, Richardson S, Austin SB, Economos CD, Rimm EB. School lunch waste among middle school students: Nutrients consumed and costs. Am J Prev Med [Internet]. 2013;44(2):114-21. Available from: http://dx.doi.org/10.1016/j.amepre.2012.09.060.

30. Figueira J. Influência da satisfação com as refeições escolares no desperdício alimentar , em crianças do 40 ano de escolaridade. 2012;1-38.

31. Bergman EA, Buergel NS, Englund TF, Femrite A. The Relationship of Meal and Recess Schedules to Plate Waste in Elementary Schools. J Child Nutr Manag [Internet]. 2004 [cited 2017 Dec 18];28(2). Available from: https://schoolnutrition.org/uploadedFiles/5_News_and_Publications/4_The_Journal_of_Child_Nutrition_and_Management/ Fall_2004/5-bergman.pdf.

32. Nicklas TA, O'Neil CE, Stuff J, Goodell LS, Liu Y, Martin CK. Validity and Feasibility of a Digital Diet Estimation Method for Use with Preschool Children: A Pilot Study. J Nutr Educ Behav. 2012;44(6):618-23.

33. Martin CK, Newton RL, Anton SD, Allen HR, Alfonso A, Han H, et al. Measurement of children's food intake with digital photography and the effects of second servings upon food intake. Eat Behav. 2007;8(2):148-56. 
34. Williamson DA, Allen HR, Martin PD, Alfonso A, Gerald B, Hunt A. Digital photography: A new method for estimating food intake in cafeteria settings. Eat Weight Disord. 2004;9(1):24-8.

35. Williamson DA, Allen HR, Martin PD, Alfonso AJ, Gerald B, Hunt A. Comparison of digital photography to weighed and visual estimation of portion sizes. J Am Diet Assoc. 2003;103(9):1139-45.

36. Giboreau A, Shwartz C, Morizet D, Meiselman HL. Measuring food waste and consumptions by children using photography. Nutrients. 2019;11(2410):10-5.

37. Christoph MJ, Loman BR, Ellison B. Developing a digital photography-based method for dietary analysis in self-serve dining settings. Appetite [Internet]. 2017;114:217-25. Available from: http://dx.doi.org/10.1016/j.appet.2017.03.050.

38. Instituto Nacional de Saúde Doutor Ricardo Jorge. Tabela da Composição de Alimentos. Serviço Nacional de Saúde. 2010.

39. Vaz CS. Restaurantes: Controlando custos e aumentado lucros. 2nd ed. Metha, editor. 2006

40. Campos V. Estudo dos desperdícios alimentares em meio escolar. 2010

41. Aragão M. Controle de aceitação de refeições em uma unidade de alimentação institucional da cidade de Fortaleza. Universidade Estadual do Ceará; 2005.

42. Liz Martins M, Rodrigues SSP, Cunha LM, Rocha A. Factors influencing food waste during lunch of fourth-grade school children. Waste Manag. 2020 Jul;113:439-46.

43. Boschini M, Falasconi L, Giordano C, Alboni F. Food waste in school canteens: A reference methodology for large-scale studies. J Clean Prod. 2018;182:1024-32. 44. Qian L, Cao B, Feng L, Wang L, Jin S. Chinese university canteens: Evidence from 9192 university students. Resour Conserv Recycl. 2021;167.

45. Lima RM. Orientações sobre Ementas e Refeitórios Escolares. Ministério da Educação - Direção Geral da Educação. 2018.

46. Garrone P, Melacini M, Perego A. Opening the black box of food waste reduction. Food Policy [Internet]. 2014;46:129-39. Available from: http://dx.doi.org/10.1016/j.foodpol.2014.03.014.

47. Ferreira J. Desperdício alimentar em duas escolas básicas do Município de Penafiel - estudo piloto [Internet]. 2012. Available from: https://repositorio-aberto.up.pt/ handle/10216/68603.

48. Liz Martins M, Cunha LM, Rodrigues SSP, Rocha A. Determination of plate waste in primary school lunches by weighing and visual estimation methods: A validation study. Waste Manag. 2014 Aug;34(8):1362-8.

49. Torres D, Faria N, Sousa N, Soares R, Guiomar S, Lobato L, et al. Manual Fotográfico de Quantificação de Alimentos. 2017.

50. Gittelsohn J, Shankar A V., Pokhrel RP, West KP. Acuracy of estimating food intake of observation. J Acad Nutr Diet. 1994;94(11):1273-7.

51. Kirks BA, Wolff HK. A comparison of methods for plate waste determinations. J Am Diet Assoc. 1985;85:328-31.

52. Thompson $\mathrm{CH}$, Head MK, Rodman SM. Factors influencing accuracy in estimating plate waste. J Am Diet Assoc. 1987;87:1219-20.

53. Comstock EM, Pierre RGS, Mackiernan YD. Measuring individual plate waste in school lunches. Visual estimation and children's ratings vs. actual weighing of plate waste. J Am Diet A. 1981;79(3):290-6.

54. Connors PL, Rozell SB. Using a visual plate waste study to monitor menu performance. J Am Diet Assoc. 2004;104:94-6.

55. Dubois S. Accuracy of visual estimates of plate waste in the dtermination of food consumption. J Am Diet Assoc. 1990;90:382-7.

56. Graves K, Shannon B. Using visual plate waste measurement to assess school lunch food behavior. J Am Diet Assoc. 1983;82.

57. Comstock EM, Symington LE. Distributions of serving sizes and plate waste in school lunches. Implications for measurement. J Am Diet Assoc. 1982;81:413-22. 